\title{
The EFIS Framework for Leveraging Agile Organizations Within Large Enterprises
}

\author{
Alexander Poth ${ }^{1(\otimes)}\left(\mathbb{D}\right.$, Mario Kottke ${ }^{1}$, Christian Heimann ${ }^{1}$, and Andreas Riel ${ }^{2}$ \\ 1 Volkswagen AG, Berliner Ring 2, 38436 Wolfsburg, Germany \\ \{alexander.poth, mario.kottke, christian.heimann\} @volkswagen. de \\ 2 Grenoble Alps University, Grenoble INP, G-SCOP, CNRS, 38031 Grenoble, France \\ andreas.riel@grenoble-inp.fr
}

\begin{abstract}
This article presents the design and application of the EFIS framework that combines four pillars to foster agile and lean working in organizations within large enterprises. These pillars constitute the empowerment of teams, the focus on products, the integration of processes, and the scaling of knowledge. The framework is designed to systematically address typical large enterprise challenges such as governance of regulation requirements and product risks. By design, EFIS is lean and nimble to make it easily adaptable to domain-specific demands within large organizations. It can be used as a stand-alone approach to establish and continuously improve lean and agile organizations, as well as in combination with existing approaches like $\mathrm{SAFe}{ }^{\circledR}$.
\end{abstract}

Keywords: Large-scaling agile $\cdot$ Agile transformation $\cdot$ Agile framework

\section{Motivation, Context and Methodology}

In the ongoing trend of the agile transformation of large companies, the set of established large-scale lean and agile frameworks has grown to a considerable number. The most established ones in practice are Scaled Agile Framework (SAFe®) [1], Large-Scale Scrum LeSS [2], Nexus [3] and Spotify [4]. Domain-specific challenges have led to specialized methodologies such as R-Scrum [5] and SafeScrum ${ }^{\circledR}$ [6] for safety. Additionally, agile organizations should address autonomy, mastery and purpose adequately [7].

Analyzing these frameworks, we identified the following shortcomings:

(1) The governance in terms of a reliable chain of accountability, responsibility, sharedresponsibility, mastery and autonomy is only vaguely addressed.

(2) Measuring the progress of the adoption of framework practices by the teams and the overall organization is not clearly covered by indicators and methods.

(3) Scaling of the framework practices without significant coaching and training efforts is not explicitly addressed. 
This paper proposes the EFIS (Empower, Focus, Integrate \& Scale) framework that aims at addressing these shortcomings from a holistic perspective. It has been designed with a bottom-up design science research approach [8] within the context of the Volkswagen Group IT. Over a timespan of five years, several building blocks have been designed, implemented, evaluated and improved. Their proven-in-use designs have been integrated in a larger framework, paying close attention to the consistency between blocks in order to ensure a holistic perspective when addressing the organizational challenges. The validation of the entire framework has been done implicitly through the validation of each building block in at least two different organizational units, as well as by measuring several teams' agile maturity progress over time.

Section 2 presents the literature and established agile frameworks. Section 3 elaborates on the architecture and application of EFIS, as well as its key characteristics. Section 4 explains how EFIS helps implementing accountability at large-scale through mastery within the scope of an agile team. Section 5 reports on how EFIS has been implemented at the Volkswagen AG, and critically evaluates success. Section 6 discusses the limitations. Finally, Sect. 7 concludes by summarizing the article's key contributions to research and practice and giving an outlook to the authors' ongoing research activities.

\section{Established Agile Frameworks and an Literature Overview}

One of the SAFe ${ }^{\circledR}[9]$ core values is "Built-In Quality", however, the focus is on product quality that is achieved through testing and/or design for quality. The quality management aspect of continuous improvement is part of the learning culture with a modified PDCA cycle [10]: the A stands for adjust instead of act in the original plan-do-checkact cycle. A core concept of LeSS [2] is to reduce organizational complexity. As part of technical excellence, LeSS focuses on testing in terms of test-driven development, thinking about testing, unit testing, as well as acceptance testing. LeSS explicitly eliminates support groups like "quality and process" as potential bottlenecks [11]. Nexus [3] is based on Scrum and defines additional accountabilities to the roles. However, it does not explicitly address governance, compliance and quality. Nexus can be seen as the enhancement of enterprise Scrum (eScrum). Scrum@Scale ${ }^{\mathrm{TM}}$ [12]: It is an agile scaling framework based on Scrum [13] and scales with the Scrum of Scrum (SoS) approach. It does not explicitly address aspects of governance and quality either. The Spotify Model is not a scaling framework by design, but rather an agile organizational building block kit [4]. Accountability is realized by the product life-cycle and features end-to-end responsibility. Furthermore, the concept of alignment enabling autonomy is used as a base for different Squads to work cooperatively on features, infrastructures or client applications. Recipes for Agile Governance in the Enterprise (RAGE) [14] are an approach focusing on making decisions repeatable and transparent. It distinguishes project, program and portfolio level. It uses Scrum and Kanban as a base for the governance extensions called recipes. Recipes are built on roles, ceremonies, artifacts, metrics and governance points. They can be combined with $\mathrm{SAFe}{ }^{\circledR}$ on the program level. For implementation RAGE offers a white paper [14] and blog posts [15]. Disciplined Agile ${ }^{\mathrm{TM}}$ (DA) [16] is a framework supporting agile and lean ways of work. The outcome is focused on solutions rather than on software only. It contains different blocks like Disciplined Agile Delivery 
(DAD) and DAE. The DAE focuses on enterprise aspects like legal and governance. Furthermore, it addresses quality in the context of software development and technical debt via the DAD process goals.

\section{The Architecture and Characteristics of EFIS}

Figure 1 shows an overview of the EFIS framework and the interaction of its individual building blocks. Internal and external regulations (right-hand side) interact with a particular organization within the enterprise (Organization@Enterprise, center). The latter's autonomous value streams implement the business domain-specific regulations and relevant standards the organization is accountable for. Stakeholders contribute knowledge and tool libraries for their domains (bottom right), serving as means of governance interaction between the organization and the enterprise. Each value stream instantiates the relevant library artifacts and enhances them if needed through its contributions via the Scale pillar (bottom left).

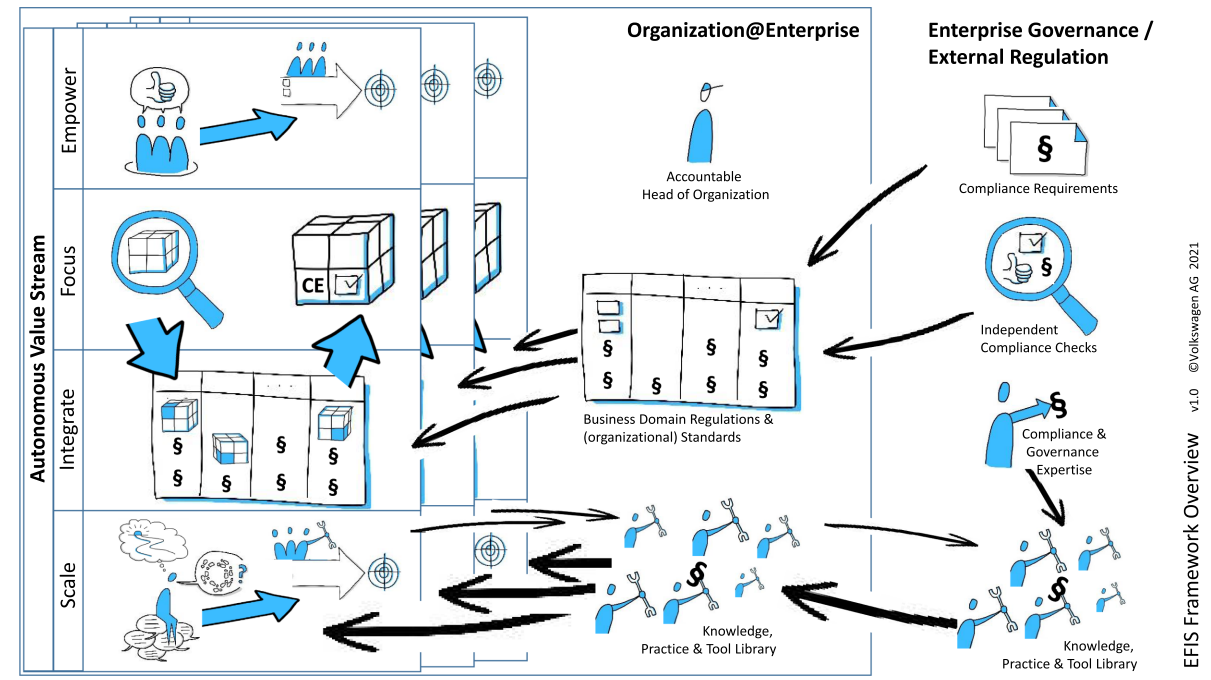

Fig. 1. The EFIS framework for autonomous value streams within an enterprise.

The operational core is the instantiation of the Focus and Integration pillars by identifying the specific product and service quality risks and integrate the related mitigation actions into the value streams' committed set of regulation and standard requirements. Once the requirements are implemented and validated systematically they assure compliant delivery outcomes. The Empower pillar (top left) continuously improves teamwork quality, for more mastery which leads to more autonomy, limiting the need for regular team supervision and evaluation.

Openness by design is a cross-cutting aspect for all pillars, and therefore not explicitly modelled. It is achieved by reducing the recommended practices to a minimum. This reduces potential conflicts with other practices and methods that can be included. 
Empower. Empowerment of product teams and their organization is achieved through systematic team development that leads to mastery, which is the prerequisite for letting them take over responsibility for their actions in an autonomous way. This, in turn, is indispensable for governing the accountability for any shipped deliverables. Empowered teams can build and improve their delivery procedures and processes independently for fast and innovative solutions. The enabling building block for systematic team empowerment is aTWQ (agile Team Work Quality) as introduced in [17].

Focus. Focus shall be set on each product/service by handling their specific risks for high quality deliveries. Each product or service comes with its business chances that also imply risks that need to be continuously investigated and updated. Systematic risk mitigation actions need to be derived and the effectiveness monitored. At the same time, the organization has to remain open for new innovative products and improvements and associated new risks. EFIS provides the building block PQR (Product Quality Risk) as introduced in [18] to address keeping focus.

Integrate. Integration of processes by interface-driven flows ensure that business domain-specific regulation and governance requirements are implemented for reliable value streams. Value streams need to be identified, including their interfaces and handover points. Regulation requirements have to be identified and derived for these value streams and their outcomes, and mapped to the hand-over points. Optionally, organizational intellectual property artifacts related to the value stream can be added to the hand-over points to ensure the property exploration within the value stream processing. Controls associated with the hand-over points enable compliance checks. One assigned individual assures the accountability for the implementation and the compliance governance of the value stream. The EFIS building block to instantiate systematic process integration is LoD (Level of Done) as introduced in [19].

Scale. Scaling of knowledge beyond individual experts and teams is achieved through encouraging knowledge self-services for organizational learning through a prosumer (producer and consumer) principle. Learning from self-services to become more mature within the business, product or service domain is encouraged. As are the sharing of any team learnings with others by building new knowledge self-services and updating existing ones. EFIS adopts the SSK (Self-Service Kit) approach as introduced in [20].

The EFIS framework establishes accountability: mature teams master their deliveries, hence they can take responsibility for their actions which enables autonomy.

\section{Leveraging Compliance Governance with EFIS}

In organizations, development and delivery processes are confronted with a growing number of regulation requirements. To avoid process complexity becoming ever larger, product- and service-related risks can be integrated into corporate governance. In an agile organization, this is feasible since the product teams are responsible for both the process and the product compliance. In EFIS, we integrated guidance and support for this incorporation process through the Product Quality Risk (PQR) building block [18]. 
EFIS builds on the shared responsibility commitment between the enterprise governance and the local organizational governance for example of a subsidiary or unit. The enterprise governance is able to delegate risk management to the local organization within a shared responsibility approach - however, the local organization risks are still part of the enterprise risk. With this approach, the process and procedure complexity can be reduced by focusing to the explicit process demands for compliance for the specific product - complex one size fit all processes are simplified to the specific products.

All procedure- and process-related compliance aspects are guided by the LoD building block [19], with the topic tasks $(\mathrm{t})$ derived from the regulations. The delivery of relevant internal organizational structures like interfaces and handover-points are modeled through the number of LoD levels. The LoD incorporates the product-specific PQR mitigation actions. The LoD and PQR together make up the core of the lean compliance approach, as depicted in Fig. 2.

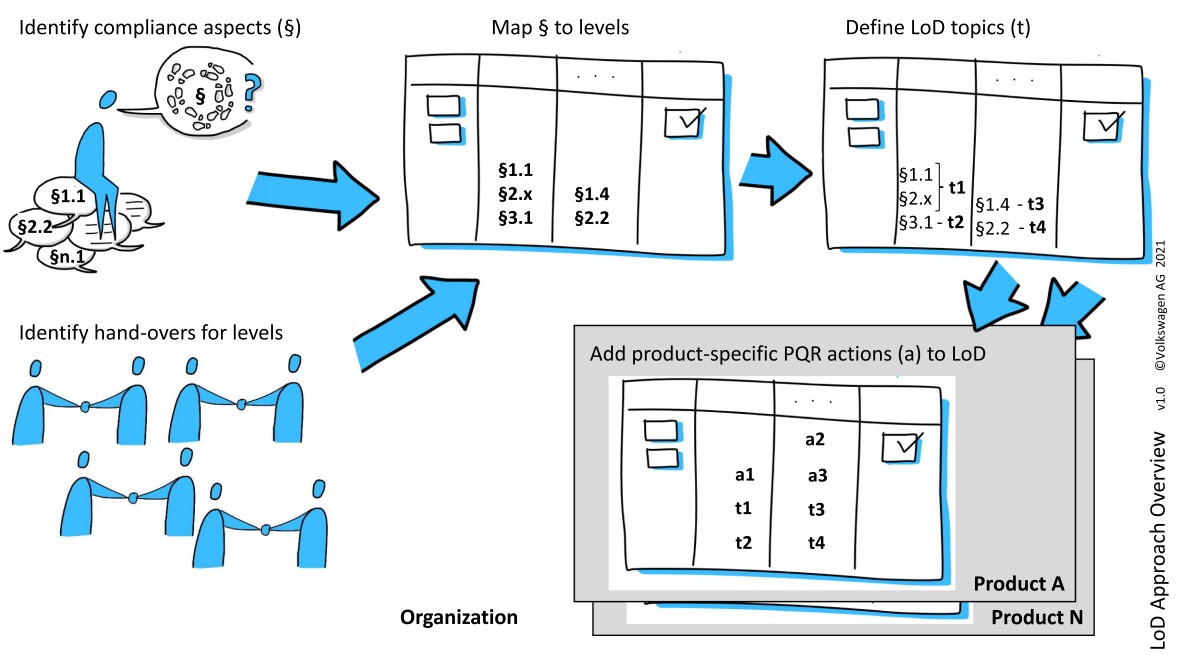

Fig. 2. A domain-specific LoD and its product instantiation.

To reduce the amount and efforts for compliance checks, the organizations' product teams have to be enabled to build compliant deliverables (entire products or services or parts of them as parts of their solutions) and assure their compliance continuously. To realize this, the teamwork quality is key: a more mature teamwork leads to better outcomes, as well as increased performance and quality of deliverables. The team maturity and mastery grows with the teams' skills and capabilities, and the organization can trust and rely on the shared responsibility principle. A highly matured team can master their products and services and work autonomously. To make team maturity transparent and help improve it, the aTWQ approach [17] is part of the empowerment pillar of the EFIS framework. 
Scaling individuals' and teams' knowledge within large-scale organizations requires encouraging employees, especially experts, to share their knowledge. In large organizations, however, not all employees know each other. Furthermore, they need to synchronize information and knowledge because the teams' product and service life-cycles are mostly independent of each other. This leads to the demand of providing expert knowledge independently of the experts' current availability. In the EFIS framework, this was realized through the SSK (Self-Service Kit) approach [20]. The essential idea behind SSKs is to share several proven-in-use practices rather than enforce particular practices as a one-size-fits-all approach. The organization and enterprise can further foster the mindset of knowledge sharing and collaboration by establishing incentives such as gamification concepts [21] for contributing to the creation and continuous improvement of SSKs.

\section{Instantiation, Evaluation and Improvement}

The Volkswagen AG has instantiated and deployed EFIS and its building blocks at different organizations and business areas. The Agile Center of Excellence (ACE) of the Volkswagen Group IT provides it in the form of an Agile Toolbox that is available to all Volkswagen Group employees. Additionally the ACE, Test \& Quality Assurance (TQA) including Quality innovation NETwork (QiNET) experts are providing coaching and facilitation services.

Some organizational units focus on all pillars, e.g., in the finance domain as well as smaller product delivery organizations such as a cloud service. Other units have been adopting selected pillars to address specific product domain demands in combination with other lean and agile methods. This is possible thanks to EFIS' modular building block architecture. Domain-specific SSK frameworks have been built to scale special matter knowledge to decentralized expert competence fields. In the Spotify model, this corresponds to a guild orchestrated by a squad of experts. However, while Spotify takes a rather structural and organizational approach, EFIS relies on a more operational and operations structuring one. Both can be fitted together, as shown in the example in Fig. 3, visualizing the instantiation adopted for the transversal expert areas like ACE, Machine Learning and Blockchain which are organized in line and virtual organizations. In this example, the Tribe Lead is accountable for the compliance of the organization and owner of the LoD. Furthermore, the Tribe Lead is interested in the continuous improvement of the organization and accountable for the SSKs. The Squads and Chapters have built and maintain both the LoD and the SSKs. They are responsible for fit for purpose in their product or service domain and the operational instantiation. The Squad Leads are accountable for the adequate instantiation of the PQR and aTWQ approach in the teams. The teams are responsible for instantiating and maintaining the EFIS artifacts.

During the EFIS evaluation period, the authors accompanied EFIS instantiations in the IT domain of finance and automotive. The acceptance and added value of EFIS is indicated by the feedbacks of people for example to SSKs and contributions to the enhancement of the EFIS framework like the LoD enhancement with LoD layers by Audi AG and Volkswagen Financial Services AG. Additionally, explicit coaching and facilitation demands are requested to the ACE. New insights and learnings were used to 
enhance the SSKs and delivery kit of the EFIS framework. Furthermore, the EFIS framework was introduced via the Agile Community of the Volkswagen AG to the community as the last step of the initial evaluation. To improve the EFIS framework continuously, feedback is collected wherever possible. Currently, the LoD layer approach in the context of shared responsibility with different governance units is under development and evaluation. For the teamwork quality pillar, the refinement of aTWQ is in progress. Due to complex IT projects, the teams need technical skills to build high quality products and services. This is addressed by team maturity for specific technologies like cloud computing.

In terms of limitations of this work, the currently known EFIS instantiations are located on different sites and legal entities in Germany. Therefore, there is a lack of information about the application of the EFIS framework in other geographical areas, especially non-European ones. However, some of the investigated teams and organizations in the German sites are highly diverse and international. Furthermore, the SSK approach fostering the autonomous instantiation of the framework and its individual building blocks limits the completeness of measurements and observations related to the actual spread and adoption levels across the entire enterprise group.

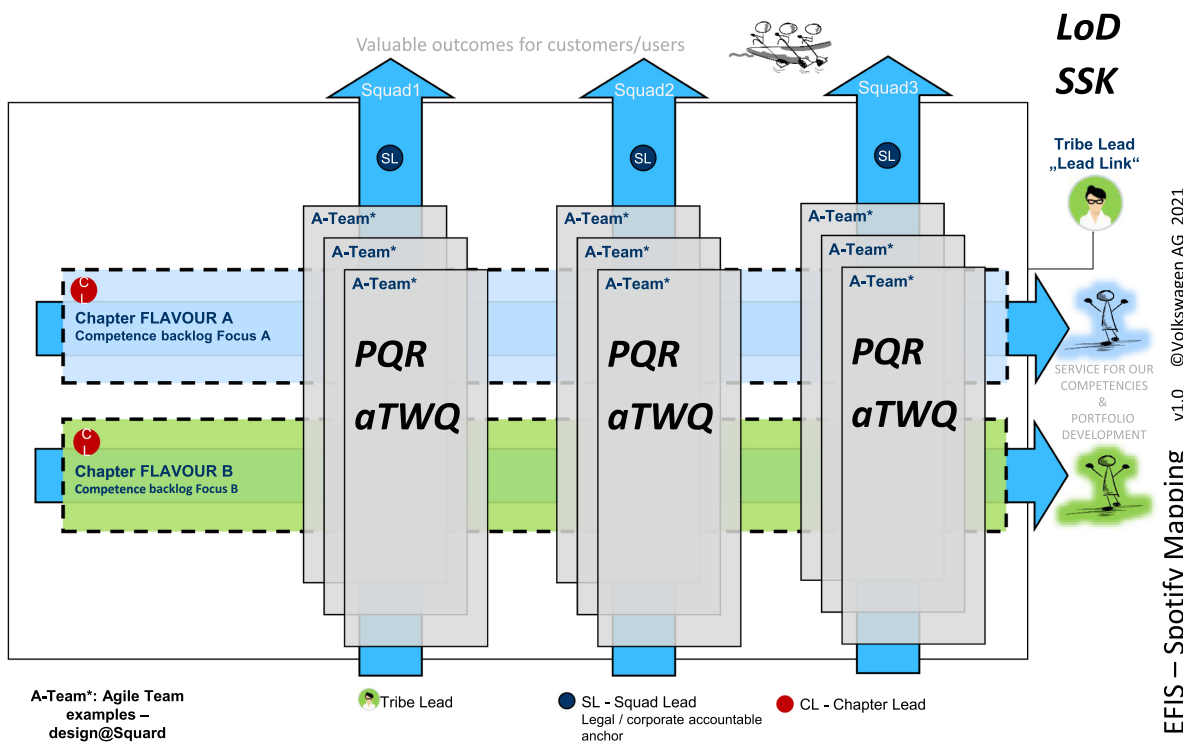

Fig. 3. Mapping of the EFIS practices to a Spotify model oriented organization.

\section{Discussion and Limitations}

EFIS is a methodology with a supporting set of tools. As SAFe ${ }^{\circledR}$ or Scrum call itself a framework the authors decide to use the term framework, too - to indicate that EFIS 
can be used stand-alone and as overlay framework on the same level as other established frameworks. An added value for product teams to the generic description of compliance integration like in $\mathrm{SAFe} \circledast[1,22]$ is that with the LoD explicit all relevant regulation and compliance requirements to the specific product are made transparent. Furthermore, all product relevant quality risks are explicit handled. Both together can be used to ensure the product and process compliance of deliveries explicit in the context and responsibility of the product team. Depending on the compliance requirements the teams can do checks manually or automated (e.g. integrated into a continuous delivery chain [23]).

As a limitation of the evaluation the instantiation in the different legal entities is that in all cases the IT applies EFIS - however this indicates that EFIS is applicable to IT organizations and also can be applied outside of the Volkswagen Group. Furthermore, no large metric set about efficiency is established for systematic monitoring and enhancement - only downloads or page views of EFIS SSKs are measured. The currently feedback driven development has to be developed to a more objective indicator and metric driven set. An idea is to use the aTWQ maturity of teams as an long-term indicator of evolvement.

\section{Conclusion and Outlook}

The EFIS framework proposed in this article provides a way to build a lean and agile environment in large-scale organizations and different domains like automotive IT or finance. Its open, modular design makes it combinable with other lean and agile practices and approaches. In particular, EFIS addresses the needs of large enterprises for systematic team development to facilitate autonomy through mastery. It achieves this through product-specific quality risk management for continuous high quality value delivery, as well as process integration for establishing delivery chains under the enterprise-compliance-governance conditions. Its fundamental underlying lever is knowledge scaling within the entire organization for continuous improvement.

The key contributions to practice can be summarized by the following aspects:

- The EFIS framework focusses on domain-specific governance in a lean and agile way, with the LoD to ensure compliance requirements and aTWQ for fostering team autonomy through mastery.

- The EFIS framework has a simple, modular structure based on four pillars and minimizes the amount of methods and practices. This is considered a key success factor for the creation and adoption of specific organizational instantiations.

- The EFIS framework can be combined with other established practices and frameworks like $\mathrm{SAFe} \circledast$ to complement them and/or to leverage transitions.

The key contributions to theory can be summarized by the following aspects:

- Identification of the gap of systematic governance and quality management by the established lean and agile practices in the context of large enterprises.

- Identification of a way to handle the accountability required by regulations by proposing a chain of accountability, responsibility, mastery and autonomy in large enterprises. 
- Demonstration that framework openness and modularity contribute to a holistic yet gradual adoption of agile and lean practices and mindset at a large scale.

Future research will address specific demands of various business domains. Furthermore, specific governance and compliance requirements have been selected for developing lean instantiation approaches and scalable patterns for the affected agile organizations within the enterprise. Additionally, systematic measures have to be established to indicate the effectiveness of the application of the EFIS framework.

\section{References}

1. SAFe®. https://www.scaledagileframework.com/. Accessed 09 July 2021

2. LeSS. https://less.works/less/framework/index. Accessed 09 July 2021

3. Nexus. https://www.scrum.org/resources/nexus-guide. Accessed 09 July 2021

4. Scaling Agile@Spotify. https://blog.crisp.se/wp-content/uploads/2012/11/SpotifyScaling. pdf. Accessed 09 July 2021

5. Fitzgerald, B., Stol, K.J., O'Sullivan, R., O’Brien, D.: Scaling agile methods to regulated environments: an industry case study. In 2013 35th International Conference on Software Engineering (ICSE), pp. 863-872. IEEE (2013)

6. Hanssen, G.K., Stålhane, T., Myklebust, T.: SafeScrum®-Agile Development of SafetyCritical Software. Springer, Heidelberg (2018). https://doi.org/10.1007/978-3-319-99334-8

7. Pink, D.H.: Drive: The Surprising Truth About What Motivates Us. Penguin, New York (2011)

8. Hevner, A.R.: A three cycle view of design science research. Scand. J. Inf. Syst. 19(2), 4 (2007)

9. SAFe ${ }^{\circledR}$ Core Values. https://www.scaledagileframework.com/safe-core-values/. Accessed 09 July 2021

10. SAFe ${ }^{\circledR}$ Continuous Learning Culture. https://www.scaledagileframework.com/continuouslearning-culture/. Accessed 15 July 2021

11. LeSS Organizational Structure. https://less.works/less/structure/organizational-structure. Accessed 15 July 2021

12. The Scrum@Scale Guide. https://www.scrumatscale.com/wp-content/uploads/2020/12/off icial-scrum-at-scale-guide.pdf. Accessed 15 July 2021

13. Scrum. https://www.scrumguides.org/. Accessed 15 July 2021

14. RAGE. https://www.cprime.com/rage/. Accessed 09 July 2021

15. Recipes for Agile Governance in the Enterprise. https://www.cprime.com/resources/blog/cat egory/agile-articles/. Accessed 23 Apr 2021

16. Disciplined Agile ${ }^{\mathrm{TM}}$ (DA). https://www.disciplinedagileconsortium.org/. Accessed 09 July 2021

17. Poth, A., Kottke, M., Riel, A.: Evaluation of agile team work quality. In: Paasivaara, M., Kruchten, P. (eds.) XP 2020. LNBIP, vol. 396, pp. 101-110. Springer, Cham (2020). https:// doi.org/10.1007/978-3-030-58858-8_11

18. Poth, A., Riel, A.: Quality requirements elicitation by ideation of product quality risks with design thinking. In: 2020 IEEE 28th International Requirements Engineering Conference (RE), pp. 238-249. IEEE (2020)

19. Poth, A., Jacobsen, J., Riel, A.: Systematic agile development in regulated environments. In: Yilmaz, M., Niemann, J., Clarke, P., Messnarz, R. (eds.) Systems, Software and Services Process Improvement: 27th European Conference, EuroSPI 2020, Düsseldorf, Germany, September 9-11, 2020, Proceedings, pp. 191-202. Springer International Publishing, Cham (2020). https://doi.org/10.1007/978-3-030-56441-4_14 
20. Poth, A., Kottke, M., Riel, A.: The implementation of a digital service approach to fostering team autonomy, distant collaboration, and knowledge scaling in large enterprises. J. Hum. Syst. Manag. 39(4), 573-588 (2020). https://doi.org/10.3233/HSM-201049

21. Festinger, L.: A theory of social comparison processes. Hum. Relat. 7(2), 117-140 (1954)

22. SAFe ${ }^{\circledR}$. Achieving Regulatory and Industry Standards Compliance with SAFe. https://www. scaledagileframework.com/achieving-regulatory-and-industry-standards-compliance-withsafe/. Accessed 14 July 2021

23. Kellogg, M., Schäf, M., Tasiran, S., Ernst, M.D.: Continuous compliance. In: 2020 35th IEEE/ACM International Conference on Automated Software Engineering (ASE), pp. 511523. IEEE (2020)

Open Access This chapter is licensed under the terms of the Creative Commons Attribution 4.0 International License (http://creativecommons.org/licenses/by/4.0/), which permits use, sharing, adaptation, distribution and reproduction in any medium or format, as long as you give appropriate credit to the original author(s) and the source, provide a link to the Creative Commons license and indicate if changes were made.

The images or other third party material in this chapter are included in the chapter's Creative Commons license, unless indicated otherwise in a credit line to the material. If material is not included in the chapter's Creative Commons license and your intended use is not permitted by statutory regulation or exceeds the permitted use, you will need to obtain permission directly from the copyright holder.

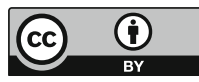

